\title{
Artigo Original / Original Paper \\ Estimativa da área foliar de Mesosphaerum suaveolens a partir de relações alométricas
}

\author{
Estimation of leaf area of Mesosphaerum suaveolens from allometric relations
}

\author{
João Everthon da Silva Ribeiro ${ }^{1,3,4}$, Jackson Silva Nóbrega ${ }^{1}$, Francisco Romário Andrade Figueiredo ${ }^{2}$, \\ Jean Telvio Andrade Ferreira ${ }^{1}$, Walter Esfrain Pereira ${ }^{1}$, Riselane de Lucena Alcântara Bruno ${ }^{1}$ \\ \& Manoel Bandeira de Albuquerque ${ }^{1}$
}

\begin{abstract}
Resumo
A determinação da área foliar é de fundamental importância em estudos ecofisiológicos que buscam entender a relação planta-ambiente. Objetivou-se com esta pesquisa determinar uma equação alométrica que permita estimar a área foliar de Mesosphaerum suaveolens a partir de medidas lineares do limbo foliar (comprimento e/ou largura). Coletaram-se 900 limbos foliares aleatórios de diferentes formas e tamanhos, em uma área de Caatinga antropizada, localizada no município de Santa Luzia, estado da Paraíba, nordeste do Brasil. Para a construção das equações alométricas utilizaram-se os seguintes modelos de regressão: linear, linear sem intercepto, quadrático, cúbico, potência e exponencial. O modelo linear sem intercepto utilizando o produto entre comprimento e largura (C.L) foi o mais indicado para estimar a área foliar de $M$. suaveolens, com maior coeficiente de determinação $\left(\mathrm{R}^{2}\right)$ e índice de concordância de Willmott $(d)$, menor erro absoluto médio (MAE), critério de informação de Akaike (AIC) e raiz do quadrado médio do erro (RQME), e índice BIAS mais próximo de zero $\left(\mathrm{R}^{2}=0,9971 ; d=0,9989 ; \mathrm{MAE}=0,422 ; \mathrm{AIC}=2094,69\right.$; $\mathrm{RQME}=0,770$; $\mathrm{BIAS}=-0,0105)$. A área foliar de $M$. suaveolens pode ser estimada satisfatoriamente pela equação $\mathrm{AF}=0,6787^{*}(\mathrm{C} . \mathrm{L})$.

Palavras-chave: alfazema-brava, biometria, Lamiaceae, método não destrutivo.
\end{abstract}

\begin{abstract}
The determination of leaf area is of fundamental importance in ecophysiological studies that seek to understand the plant-environment relationship. The objective of this research was to determine an allometric equation to estimate the leaf area of Mesosphaerum suaveolens from linear measures of leaf blade (length and/or width). There were collected 900 random leaf limbs of different shapes and sizes, in an anthropic area of caatinga, located in the municipality of Santa Luzia, state of Paraíba, Northeast of Brazil. For the construction of the allometric equations the following regression models were used: linear, linear without intercept, quadratic, cubic, power, and exponential. The linear model without intercept using the product between length and width (C.L) was the most recommended to estimate the leaf area of M. suaveolens, with greater coefficient of determination $\left(\mathrm{R}^{2}\right)$ and Willmott concordance index $(d)$, lesser mean absolute error (MAE), Akaike information criterion (AIC) and root-mean-square error $(\mathrm{RQME})$, and the closer the BIAS ratio is to zero $\left(\mathrm{R}^{2}=0.9971 ; \mathrm{d}=0.9989 ; \mathrm{MAE}=0.422 ; \mathrm{AIC}=\right.$ 2094.69; $\mathrm{RQME}=0.770 ; \mathrm{BIAS}=-0.0105)$. The leaf area of $M$. suaveolens can be estimated satisfactorily by equation $\mathrm{AF}=0.6787^{*}(\mathrm{C} . \mathrm{L})$.
\end{abstract}

Key words: alfazema-brava, biometry, Lamiaceae, non-destructive method.

\footnotetext{
${ }^{1}$ Universidade Federal da Paraíba, Centro de Ciências Agrárias, Rod. BR-079, Campus II, 58397-000, Areia, PB, Brasil.

${ }^{2}$ Universidade Federal Rural do Semi-Árido, Av. Francisco Mota-572, Bairro Costa e Silva, 59625-900, Mossoró, RN, Brasil

${ }^{3}$ ORCID: <https://orcid.org/0000-0002-1937-0066>

${ }^{4}$ Autor para correspondência: j.everthon@hotmail.com
} 


\section{Introdução}

Mesosphaerum suaveolens (L.) Kuntze é uma espécie anual pertencente à família Lamiaceae, com porte de 0,50 a 1,90 podendo atingir até 2,5 $\mathrm{m}$ de altura, de acordo com o ambiente (Raizada 2006). Sua ocorrência é comum em ambientes antropizados, como por exemplo, em beira de estradas, áreas de pastagens, cultivos perenes e anuais (Maia et al. 2008). Apresenta distribuição geográfica em regiões tropicais e subtropicais, principalmente na América do Sul, África Ocidental e norte da Nigéria. No Brasil é registrada em todas as regiões do território nacional e destaca-se pelo alto valor na medicina popular, principalmente na Região Nordeste, sendo conhecida popularmente como cheirosa, alfavacão, metrasto-graçú, melissade-pison, pataquera, betônica-brava, betôniabranca, bamburral, sambacoité, alfazema-brava, salva-limão, mentrasto-do-grande, alfavaca-decaboclo, alfazema-de-caboclo e chá-de-frança (Lorenzi \& Matos 2002).

A parte aérea das plantas medicinais é a principal matéria prima para fabricação de produtos fitoterápicos, principalmente as folhas, onde é encontrado maior quantidade de compostos bioativos (Koche et al. 2010). Nas folhas de M. suaveolens estão presentes quantidades significativas de alcaloides, flavonoides, saponinas, taninos e terpenos, que são empregados nos tratamentos de bronquite, doenças de pele, problemas respiratórios em geral, febre, distúrbios no sistema digestivo, ação antibacteriana, antifúngica e também como inseticida, contra Aedes aegypti ( $4^{\circ}$ estágio) (Falcão \& Menezes 2003; Gómez-Peralta et al. 2009; Satish et al. 2010; Chatri et al. 2014).

Diante da importância dessa espécie, estudos ecofisiológicos são importantes para avaliar o crescimento, desenvolvimento e reprodução da planta. Dentre esses estudos, a estimativa da área foliar vem se destacando, considerando-se como o parâmetro de maior importância para o desenvolvimento de plantas (Taiz et al. 2017). Inúmeros estudos foram realizados para estimativa da área foliar de espécies medicinais, como Jatropha curcas L. (Pompelli et al. 2012), Rosa hybrida L. (Gao et al. 2012), Solanum melongela L. (Hinnah et al. 2014), Calotropis procera (Aiton) W.T. Aiton. (Ferreira et al. 2015), Brassica napus L. (Tartaglia et al. 2016), Crotalaria juncea L. (Carvalho et al. 2017a), Plectranthus ornatus Codd. (Silva et al. 2017), e Commelina difusa
Burm.f. (Carvalho et al. 2017b).

Este parâmetro é utilizado como medida indicativa de crescimento e produtividade de vegetais, devido as folhas serem responsáveis pelas trocas gasosas, atuando diretamente na eficiência fotossintética, transpiração, quantidade de nutrientes e produção de biomassa (Jadoski et al. 2012; Schmildt et al. 2014).

Existem diferentes métodos utilizados para determinação da área foliar, sendo classificados como diretos e indiretos, destrutivos e não destrutivos (Peksen 2007; Sousa et al. 2015). Os métodos diretos (destrutivos), geralmente são simples e precisos, porém demandam maior tempo e mão de obra, bem como provocam a destruição das folhas (Lu et al. 2004; Grecco et al. 2011), tornando-se inviável em espécies ameaçadas de extinção, de pequeno porte e em estágios de vida que não suportam a retirada dos limbos foliares. $\mathrm{O}$ uso de equações alométricas para estimativa da área foliar a partir do comprimento e largura dos limbos foliares é um método não destrutivo (método indireto) tão eficiente quanto os métodos destrutivos, sendo mais simples e prático, mesmo sob diferentes condições das plantas, permitindo múltiplas avaliações durante todo o ciclo dos indivíduos (Pompelli et al. 2012; Mota et al. 2014).

Diante disso, o objetivo da pesquisa foi construir uma equação alométrica (método não destrutivo) que determine a área foliar de $M$. suaveolens a partir de medidas lineares dos limbos foliares.

\section{Material e Métodos}

A pesquisa foi realizada em uma área de Caatinga antropizada, no município de Santa Luzia, localizado na microrregião do Seridó Ocidental Paraibano e mesorregião da Borborema, do estado da Paraíba, nordeste do Brasil (6 62'19'S, $36^{\circ} 55^{\prime} 08^{\prime}$ W) (Fig. 1). A região apresenta temperatura entre 25 e $28{ }^{\circ} \mathrm{C}$ (Damasceno et al. 2010), altitude de 299 metros e média pluviométrica anual de $550,5 \mathrm{~mm}$. O clima é semiárido quente $(B S h)$, segundo a classificação Köppen, com irregularidades no regime pluviométrico durante todo o ano.

Coletaram-se aleatoriamente 900 limbos foliares com formatos e tamanhos diferentes em 50 indivíduos de $M$. suaveolens, sendo as folhas saudáveis, livres de danos, pragas, doenças e, ou, fatores abióticos (Lucena et al. 2011). Posteriormente, os limbos foliares foram 

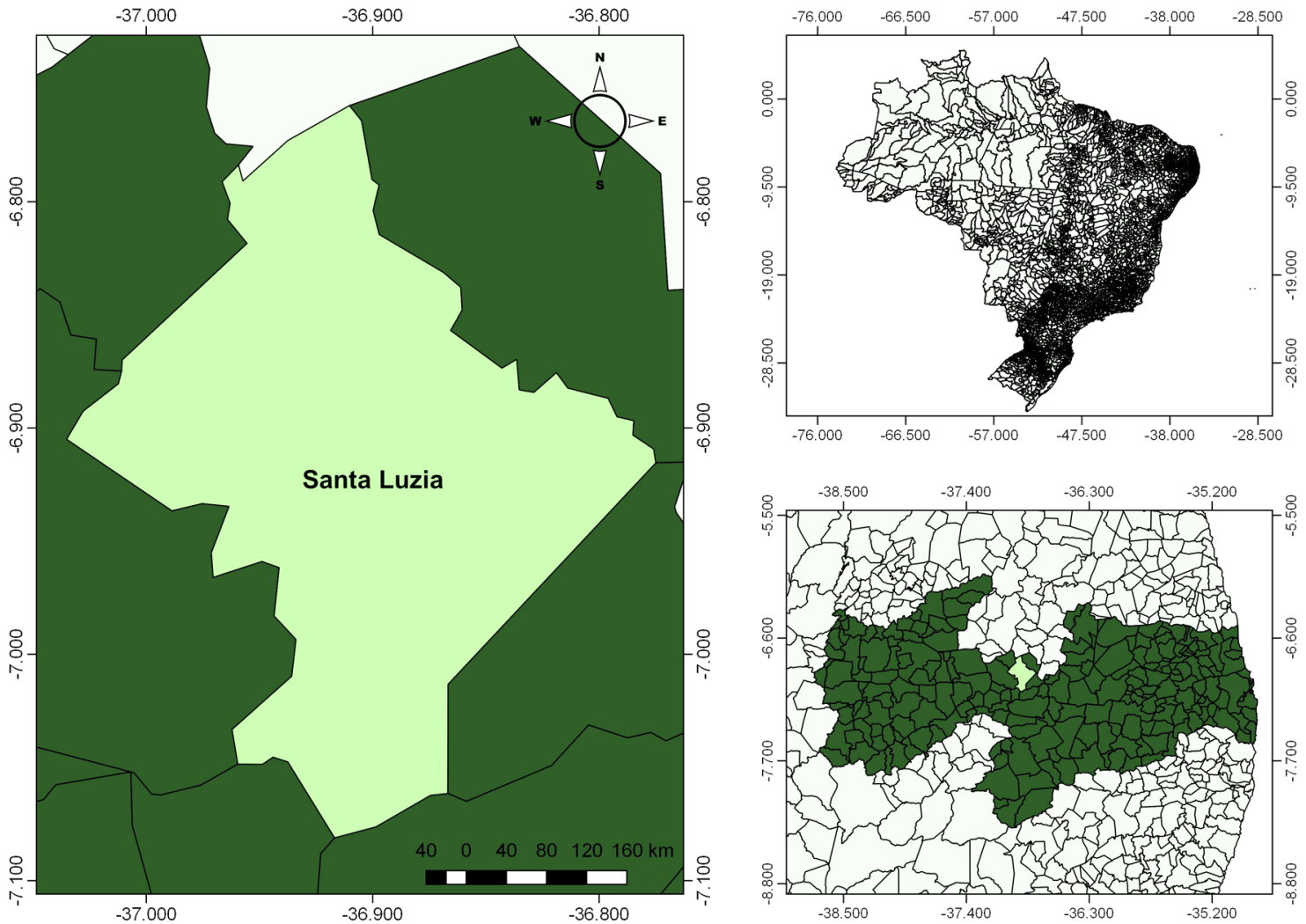

Figura 1 - Localização geográfica do município de Santa Luzia, estado da Paraíba, nordeste do Brasil.

Figure 1 - Geographic location of municipality of Santa Luzia, state of Paraíba, Northeast of Brazil.

armazenados e acondicionados em sacos plásticos, e encaminhados ao Laboratório de Ecologia Vegetal, pertencente a Universidade Federal da Paraíba, Campus II. Em cada limbo, mensurouse o comprimento máximo $(\mathrm{C})$ e largura máxima (L) (Fig. 2), através de uma régua graduada em milímetros. A partir dos valores obtidos do comprimento e largura, calculou-se o produto entre esses parâmetros (C.L). Para determinação da área foliar real (AF), os limbos foram digitalizados a partir de um scanner de mesa (Canon, P-215II), e as imagens foram contrastadas e analisadas com uso do Software ImageJ ${ }^{\circledR}$ (Ribeiro et al. 2018).

A partir dos dados de comprimento (C), largura (L), produto entre comprimento e largura (C.L), e área foliar real (AF) dos 900 limbos foliares, realizou-se uma análise descritiva dos dados e calcularam-se os valores mínimo e máximo; média, mediana, variância, amplitude total, desvio padrão, erro padrão e coeficiente de variação, para cada variável.

Para a construção das equações alométricas, empregou-se o uso dos modelos de regressão: linear

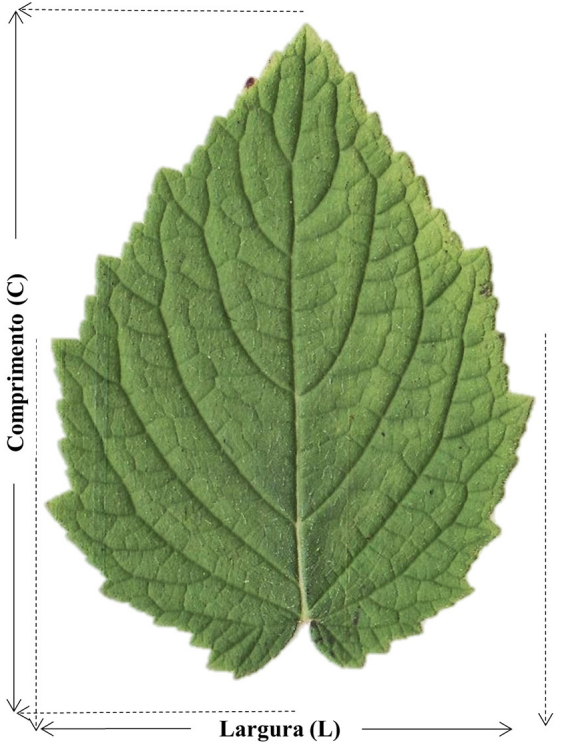

Figura 2 - Dimensões lineares do limbo foliar (comprimento e largura) de M. suaveolens utilizados na estimativa da área foliar.

Figure 2 - Linear dimensions of the leaf blade (length and width) of M. suaveolens used in the estimation of the leaf area. 
simples, linear sem intercepto, quadrático, cúbico, potência e exponencial. A descrição dos modelos utilizados está apresentada na Tabela 1. Para escolha da melhor equação que estime satisfatoriamente a área foliar de $M$. suaveolens a partir de dimensões lineares das folhas, utilizaram-se os critérios de maior valor do coeficiente de determinação $\left(R^{2}\right)$ e índice de concordância de Willmott (d) (Willmott et al. 1985); menor erro absoluto médio (MAE), critério de informação de Akaike (AIC) (Floriano et al. 2006) e raiz do quadrado médio do erro (RQME) (Janssen \& Heuberger 1995; Xue et al. 2004); e índice BIAS mais próximo de zero (Leite \& Lima 2002). As análises estatísticas foram realizadas no software $R \circledR$ v.3.5.1 (R Core Team 2018).

Tabela 1 - Modelos utilizados na estimativa da área foliar de M. suaveolens.

Table 1 - Models used to estimate the leaf area of M. suaveolens.

\begin{tabular}{|c|c|}
\hline Modelo & Descrição do modelo \\
\hline Linear & $\mathrm{AF}=\mathrm{a}+\mathrm{b}^{*} \mathrm{C}$ \\
\hline Linear & $\mathrm{AF}=\mathrm{a}+\mathrm{b} * \mathrm{~L}$ \\
\hline Linear & $\mathrm{AF}=\mathrm{a}+\mathrm{b} *(\mathrm{C} . \mathrm{L})$ \\
\hline Linear sem intercepto $(0,0)$ & $\mathrm{AF}=\mathrm{b} *(\mathrm{C} . \mathrm{L})$ \\
\hline Quadrático & $\mathrm{AF}=\mathrm{a}^{*} \mathrm{C}^{2}+\mathrm{b}^{*} \mathrm{C}+\mathrm{c}$ \\
\hline Quadrático & $\mathrm{AF}=\mathrm{a}^{*} \mathrm{~L}^{2}+\mathrm{b} * \mathrm{~L}+\mathrm{c}$ \\
\hline Quadrático & $\mathrm{AF}=\mathrm{a}^{*}(\mathrm{C} . \mathrm{L})^{2}+\mathrm{b}^{*}(\mathrm{C} . \mathrm{L})+\mathrm{c}$ \\
\hline Cúbico & $\mathrm{AF}=\mathrm{a}^{*} \mathrm{C}^{3}+\mathrm{b}^{*} \mathrm{C}^{2}+\mathrm{c}^{*} \mathrm{C}+\mathrm{d}$ \\
\hline Cúbico & $\mathrm{AF}=\mathrm{a}^{*} \mathrm{~L}^{3}+\mathrm{b}^{*} \mathrm{~L}^{2}+\mathrm{c}^{*} \mathrm{~L}+\mathrm{d}$ \\
\hline Cúbico & $\mathrm{AF}=\mathrm{a}^{*}(\mathrm{C} . \mathrm{L})^{3}+\mathrm{b}^{*}(\mathrm{C} . \mathrm{L})^{2}+\mathrm{c}^{*}(\mathrm{C} . \mathrm{L})+\mathrm{d}$ \\
\hline Potência & $\mathrm{AF}=\mathrm{a}^{*} \mathrm{C}^{\mathrm{b}}$ \\
\hline Potência & $\mathrm{AF}=\mathrm{a}^{*} \mathrm{~L}^{\mathrm{b}}$ \\
\hline Potência & $\mathrm{AF}=\mathrm{a}^{*}(\mathrm{C} \cdot \mathrm{L})^{\mathrm{b}}$ \\
\hline Exponencial & $\mathrm{AF}=\mathrm{a}^{*} \mathrm{~b}^{\mathrm{C}}$ \\
\hline Exponencial & $\mathrm{AF}=\mathrm{a}^{*} \mathrm{~b}^{\mathrm{L}}$ \\
\hline Exponencial & $\mathrm{AF}=\mathrm{a}^{*} \mathrm{~b}^{(\mathrm{C} . \mathrm{L})}$ \\
\hline
\end{tabular}

\section{Resultados e Discussão}

A análise descritiva dos dados obtidos a partir dos limbos foliares de M. suaveolens com os valores mínimo, máximo, média, mediana, variância, amplitude total, desvio padrão, erro padrão e coeficiente de variação, estão apresentados na Tabela 2. Os valores do comprimento das folhas (C) variaram de 0,83 a 10,69 cm, com média de 3,79 $\mathrm{cm}$; a largura dos limbos foliares apresentou média de 2,54, com valores que variaram de 0,44 a 7,89 $\mathrm{cm}$; o produto entre comprimento e largura (C.L) variou de 0,36 a $80,48 \mathrm{~cm}^{2}$, com média de 13,45; e a área foliar real (AF) apresentou valores médios de $9,05 \mathrm{~cm}^{2}$, que variaram de 0,28 a $57,11 \mathrm{~cm}^{2}$ (Tab. 2).
De acordo com a variabilidade dos dados, os menores coeficientes de variação foram observados nas dimensões do comprimento e largura $(58,61 \mathrm{e}$ $69,26 \%$, respectivamente), e a maior variabilidade foi constatada nos parâmetros do produto entre comprimento e largura $(88,66 \%)$, e na área foliar real $(90,74 \%)$ (Tab. 2). Valores elevados de amplitude total, desvio padrão e coeficiente de variação são de extrema importância em estudos que envolvem o uso de modelos de regressão na estimativa da área foliar. Quanto maior a variabilidade dos dados avaliados, melhor será a representatividade dos modelos, pois assim permitirá a utilização em diferentes estádios fenológicos da planta. Diante disso, o número de limbos foliares utilizados no presente estudo (900 limbos) é ideal para construir 
Tabela 2 - Mínimo, máximo, média, mediana, variância, amplitude total, desvio padrão, erro padrão e coeficiente de variação (C.V.) para comprimento, largura, produto entre comprimento e largura, e área foliar real de 900 limbos foliares de $M$. suaveolens.

Table 2 - Minimum, maximum, mean, median, variance, total amplitude, standard deviation, standard error and coefficient of variation (C.V.) for length, width, product between length and width, and real leaf area of $900 \mathrm{M}$. suaveolens leaf blades.

\begin{tabular}{lcccc}
\hline Estatística descritiva & Comprimento & Largura & Produto (C.L) & Área foliar \\
\hline Mínimo & 0,83 & 0,44 & 0,36 & 0,28 \\
Máximo & 10,69 & 7,89 & 80,48 & 57,11 \\
Média & 3,79 & 2,54 & 13,45 & 9,05 \\
Mediana & 2,92 & 1,85 & 5,29 & 3,58 \\
Variância & 4,95 & 3,09 & 299,87 & 140,20 \\
Amplitude Total & 9,86 & 7,45 & 80,12 & 56,83 \\
Desvio Padrão & 2,22 & 1,75 & 17,31 & 11,84 \\
Erro Padrão & 0,07 & 0,06 & 0,57 & 0,39 \\
C.V. (\%) & $58,61 \%$ & $69,26 \%$ & $88,66 \%$ & $90,74 \%$ \\
\hline
\end{tabular}

modelos que permitam estimar a área foliar da espécie em função de dimensões das folhas. A ampla variabilidade registrada para o produto (C.L) e área foliar real (AF) também foi encontrada em outros estudos (Toebe et al. 2012; Francisco et al. 2014; Schmildt et al. 2015, 2016a; Leite et al. 2017; Ribeiro et al. 2018).

A distribuição percentual dos 900 limbos foliares de M. suaveolens em relação as faixas de tamanho, estão apresentados na Tabela 3. Observase que $54,6 \%$ da área foliar real está dentro da faixa de 0,25 a $4,0 \mathrm{~cm}^{2}$, evidenciando que a maioria dos limbos foliares desta espécie são de tamanho pequeno.
$\mathrm{Na}$ Tabela 4, observa-se as equações alométricas propostas a partir dos modelos de regressão relacionando a área foliar real (AF) em função de medidas lineares do limbo foliar (C, L e C.L). Observou-se que todas as equações propostas podem ser utilizadas para estimar de maneira satisfatória a área foliar de M. suaveolens, com altos valores dos coeficientes de determinação $\left(\mathrm{R}^{2}\right.$ $>0,90)$, confirmando que pelo menos $90 \%$ das variações nas áreas foliares reais da espécie foram explicadas pelas equações, a partir de dimensões lineares dos limbos foliares.

As equações que utilizaram o produto entre comprimento e largura (C.L) apresentaram critérios

Tabela 3 - Distribuição percentual da área foliar real (AF) de 900 limbos foliares de M. suaveolens em diferentes faixas de tamanho.

Table 3 - Percentage distribution of the real leaf area (AF) of 900 leaf blades of M. suaveolens in different size ranges.

\begin{tabular}{cc}
\hline Área foliar $\left(\mathbf{c m}^{2}\right)$ & $\mathbf{( \% )}$ \\
\hline$[0,25-4,0]$ & 54,6 \\
{$[4,01-8,0]$} & 16,6 \\
{$[8,01-16,0]$} & 10,2 \\
{$[16,01-24,0]$} & 5,0 \\
{$[24,01-32,0]$} & 5,2 \\
{$[32,01-40,0]$} & 5,2 \\
{$[40,01-58,0]$} & 3,2 \\
\hline
\end{tabular}


Tabela 4 - Modelos, equações, coeficiente de determinação $\left(\mathrm{R}^{2}\right)$, critério de informação de Akaike (AIC), raiz do quadrado médio do erro (RQME), erro absoluto médio (MAE), índice de concordância de Willmott (d) e índice BIAS em função de medidas lineares das folhas de M. suaveolens.

Table 4 - Models, equations, coefficient of determination $\left(R^{2}\right)$, Akaike information criterion (AIC), root-mean-square error (RQME), mean absolute error (MAE), Willmott concordance index $(d)$ and the BIAS ratio as a function of linear measurements of leaves of $M$. suaveolens.

\begin{tabular}{lccccccc}
\hline Modelo & $\mathbf{R}^{2}$ & AIC & RQME & MAE & $\boldsymbol{d}$ & BIAS & Equação \\
\hline Linear & 0,9058 & 4880,71 & 3,630 & 2,701 & 0,9747 & $-0,0782$ & $\mathrm{AF}=-10,172+5,064^{*} \mathrm{C}$ \\
Linear & 0,9587 & 4139,75 & 2,405 & 1,820 & 0,9893 & $-0,0188$ & $\mathrm{AF}=-7,683+6,591^{*} \mathrm{~L}$ \\
Linear & 0,9955 & 2149,78 & 0,796 & 0,442 & 0,9988 & $-0,0166$ & $\mathrm{AF}=-0,1256+0,6822^{*}(\mathrm{C} . \mathrm{L})$ \\
Linear $(0,0)$ & 0,9971 & 2094,69 & 0,770 & 0,422 & 0,9989 & $-0,0105$ & $\mathrm{AF}=0,6787 *(\mathrm{C} . \mathrm{L})$ \\
Quadrático & 0,9732 & 3751,42 & 1,936 & 1,051 & 0,9931 & 0,0451 & $\mathrm{AF}=0,658^{*} \mathrm{C}^{2}-1,524^{*} \mathrm{C}+2,098$ \\
Quadrático & 0,9927 & 2579,35 & 1,010 & 0,608 & 0,9981 & 0,0855 & $\mathrm{AF}=0,7451^{*} \mathrm{~L}^{2}+1,0564^{*} \mathrm{~L}-0,7350$ \\
Quadrático & 0,9956 & 2161,62 & 0,802 & 0,435 & 0,9988 & 0,0387 & $\mathrm{AF}=0,0004^{*}(\mathrm{C} . \mathrm{L})^{2}+0,6545^{*}(\mathrm{C} . \mathrm{L})+0,0203$ \\
Cúbico & 0,9731 & 3753,37 & 1,936 & 1,048 & 0,9931 & 0,0206 & $\mathrm{AF}=0,0011^{*} \mathrm{C}^{3}+0,6396^{*} \mathrm{C}^{2}-1,4403^{*} \mathrm{C}+1,9931$ \\
Cúbico & 0,9927 & 2581,16 & 1,010 & 0,606 & 0,9981 & 0,0236 & $\mathrm{AF}=-0,0024^{*} \mathrm{~L}^{3}+0,7734^{*} \mathrm{~L}^{2}+0,9654^{*} \mathrm{~L}-0,6583$ \\
Cúbico & 0,9957 & 2118,24 & 0,782 & 0,425 & 0,9988 & $-0,0124$ & $\mathrm{AF}=-0,00001^{*}(\mathrm{C} . \mathrm{L})^{3}+0,0024^{*}(\mathrm{C} . \mathrm{L})^{2}+$ \\
Potência & 0,9730 & 3760,47 & 1,948 & 1,056 & 0,9931 & 0,0860 & $0,6044 *(\mathrm{C} . \mathrm{L})+0,2006$ \\
Potência & 0,9927 & 2579,37 & 1,011 & 0,600 & 0,9981 & $-0,0195$ & $\mathrm{AF}=0,2963^{*} \mathrm{C}^{2,2507}$ \\
Potência & 0,9956 & 2118,77 & 0,782 & 0,434 & 0,9988 & 0,0164 & $\mathrm{AF}=1,203^{*} \mathrm{~L}^{1,840}$ \\
Exponencial & 0,9488 & 4422,57 & 2,814 & 2,019 & 0,9845 & $-0,6490$ & $\mathrm{AF}=0,6229^{*}(\mathrm{C} . \mathrm{L})^{1,0225}$ \\
Exponencial & 0,9596 & 4240,73 & 2,544 & 2,094 & 0,9874 & $-0,6743$ & $\mathrm{AF}=1,699^{*} 1,428^{\mathrm{C}}$ \\
Exponencial & 0,9488 & 5189,15 & 2,544 & 2,094 & 0,9874 & $-0,6743$ & $\mathrm{AF}=2,323^{*} 1,525^{\mathrm{L}}$ \\
\hline
\end{tabular}

satisfatórios para os modelos de regressão em comparação aos que empregaram o comprimento (C) ou largura (L) (Tab. 4), proporcionando melhor ajuste dos modelos (Cardozo et al. 2014; Sachet et al. 2015; Schmildt et al. 2016b; Oliveira et al. 2017; Lucena et al. 2018), exceto para o modelo exponencial onde os melhores índices foram encontrados quando utiliza-se apenas a largura das folhas (Kumar 2009; Figueiredo et al. 2012; Silva et al. 2017).

Em relação aos critérios utilizados para escolha da melhor equação que estime satisfatoriamente a área foliar de M. suaveolens em função de medidas foliares, o modelo linear sem intercepto utilizando o produto entre comprimento e largura (C.L) obteve os maiores valores de $\mathrm{R}^{2}$ e $d(0,9971$ e 0,9989 , respectivamente), menores valores de MAE $(0,422)$, AIC $(2094,69)$ e RQME $(0,770)$, e BIAS mais próximo de zero $(-0,0105)$ (Tab. 4). Sendo assim, a área foliar de $M$. suaveolens pode ser estimada através da equação $\mathrm{AF}=0.6787 *(\mathrm{C} . \mathrm{L})$, que corresponde a $67,87 \%$ do produto (C.L). Este modelo também foi indicado para estimativa da área foliar de outras espécies medicinais, como por exemplo Ageratum conyzoides L. [AF = 0,6789*(C.L)] (Bianco et al. 2008), Ziziphus joazeiro Mart. $[\mathrm{AF}=0,7931 *(\mathrm{C} . \mathrm{L})]$ (Maracajá et al. 2008), Combretum leprosum Mart. [AF = 0,7103*(C.L)] (Candido et al. 2013), Capsicum annuum L. $[\mathrm{AF}=0,57 *(\mathrm{C} . \mathrm{L})]$ (Padrón et al. 2016), Crotalaria juncea L. [AF $=0,7390 *(C . L)]$ (Carvalho et al. 2017a).

De acordo com a equação selecionada para estimar a área foliar da espécie $\left[\mathrm{AF}=0.6787^{*}(\mathrm{C} . \mathrm{L})\right]$, houve pouca dispersão dos dados em relação a reta com a curva ajustada, indicando satisfatoriamente a precisão do modelo escolhido (Fig. 3). Diante disso, a estimativa da área foliar de $M$. suaveolens a partir da equação $\mathrm{AF}=0.6787^{*}(\mathrm{C} . \mathrm{L})$ apresentou uma alta correlação positiva com a área foliar real, em que o 


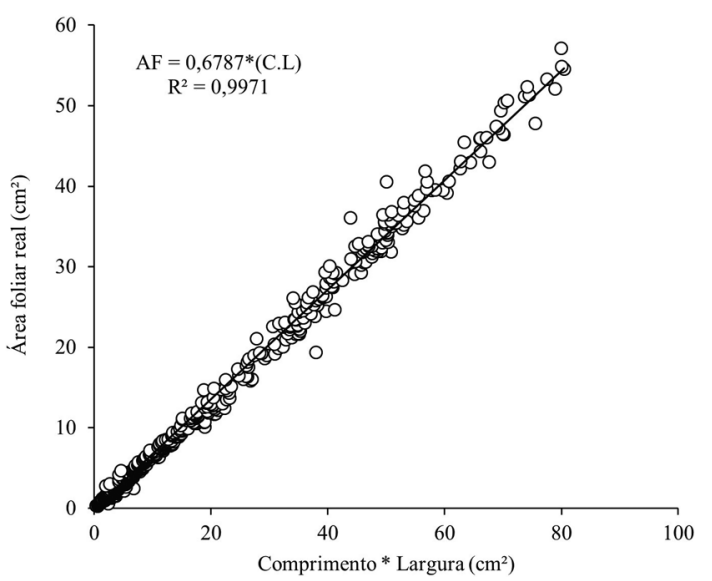

Figura 3 - Relação entre área foliar real (AF) de $M$. suaveolens em função do produto (C.L) dos limbos foliares, pela equação indicada para estimar a área foliar $[\mathrm{AF}=0,6787 *(\mathrm{C} . \mathrm{L})]$.

Figure 3 - Relation between real leaf area $(\mathrm{AF})$ of $M$. suaveolens as a function of the product (C.L) of leaf blades, by the equation indicated to estimate the leaf area $[\mathrm{AF}=0.6787 *(\mathrm{C} . \mathrm{L})]$.

coeficiente de determinação $\left(\mathrm{R}^{2}\right)$ foi de 0,9955 entre estes parâmetros (Fig. 4). Dessa forma, área foliar de $M$. suaveolens pode ser estimada com precisão a partir do método não destrutivo, empregando-se o produto entre comprimento e largura (C.L) dos limbos foliares. A equação $\mathrm{AF}=0,6787^{*}(\mathrm{C} . \mathrm{L})$ estima de forma satisfatória a área foliar de $M$. suaveolens de forma prática e rápida.

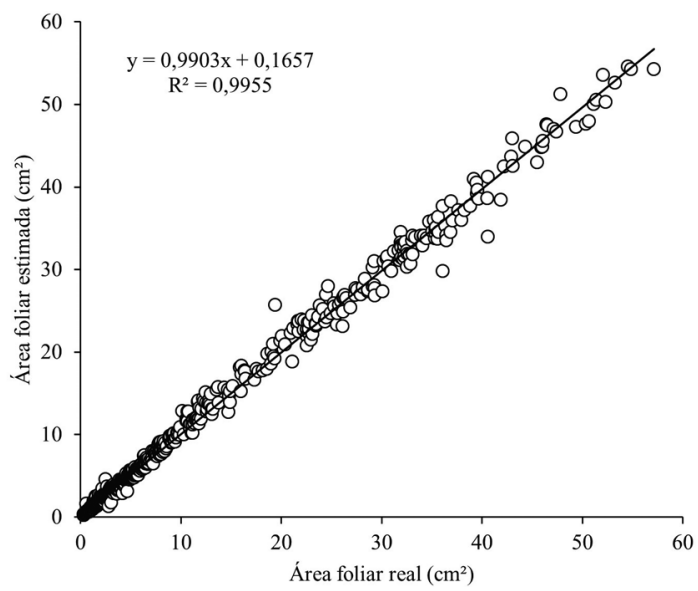

Figura 4 - Relação entre área foliar real e área foliar estimada de $M$. suaveolens a partir da equação de regressão $\mathrm{AF}=0,6787 *(\mathrm{C} . \mathrm{L})$.

Figure 4 - Relation between real leaf area and estimated leaf area of $M$. suaveolens by the regression equation $\mathrm{AF}=0.6787 *(\mathrm{C} . \mathrm{L})$.

\section{Agradecimentos}

O presente trabalho foi realizado com apoio da Coordenação de Aperfeiçoamento de Pessoal de Nível Superior - Brasil (CAPES) - Código de Financiamento 001.

\section{Referências}

Bianco S, Bianco MS \& Carvalho LB (2008) Estimativa da área foliar de Ageratum conyzoides usando dimensões lineares do limbo foliar. Acta Scientiarum. Agronomy 30: 519-523.

Candido WS, Coelho MFB, Maia SSS, Cunha CSM \& Silva RCP (2013) Modelo para estimar a área foliar de Combretum leprosum Mart. Acta Agronomica 62: 37-41.

Cardozo NP, Parreira MC, Panosso AR \& Volpe CA (2014) Modelagem da área foliar de duas cultivares de amendoim em função das dimensões lineares dos folíolos. Bioscience Journal 30: 101-107.

Carvalho JO, Toebe M, Tartaglia FL, Bandeira CT \& Tambara AL (2017a) Leaf area estimation from linear measurements in different ages of Crotalaria juncea plants. Anais da Academia Brasileira de Ciências 89: 1851-1868.

Carvalho LB, Alves EA \& Bianco S (2017b) Nondestructive model to predict Commelina diffusa leaf area. Planta Daninha 35: 1-5.

Chatri M, Baktiar A \& Adnadi P (2014) Chemical components of essential oils of the leaves of Hyptis suaveolens (L.) Poit. from Indonesia. American Journal of Research Communication 2: 30-38.

Damasceno MM, Souto JS \& Souto PC (2010) Etnoconhecimento de espécies forrageiras no semiárido da Paraíba, Brasil. Engenharia Ambiental 7: 219-228.

Falcão DC \& Menezes FS (2003) Revisão etnofarmacológica, farmacológica e química do gênero Hyptis. Revista Brasileira de Farmácia 84: 69-74.

Ferreira MLA, Silva RA, Pinto MSC, Silva EA \& Silva FJ (2015) Determinação da área foliar da flor de seda (Calotropis procera) no sertão paraibano. Agropecuária Científica no Semiárido 11: 53-55.

Figueiredo ES, Santos ME \& Garcia A (2012) Modelos de determinação não destrutivo da área foliar do feijoeiro comum (Phaseolus vulgaris L.). Nucleus 9: 79-84.

Floriano EP, Müller I, Finger CAG \& Schneider PR (2006) Ajuste e seleção de modelos tradicionais para série temporal de dados de altura de árvores. Ciência Florestal 16: 177-199.

Francisco JP, Diotto AV, Folegatti MV, Silva LDB \& Piedade SMS (2014) Estimativa da área foliar do abacaxizeiro cv. Vitória por meio de relações alométricas. Revista Brasileira de Fruticultura 36: 285-293. 
Gao M, Van Der Heijden G, Vos J, Eveleens BA \& Marcelis LFM (2012) Estimation of leaf area for large scale phenotyping and modeling of rose genotypes. Scientia Horticulturae 138: 227-234.

Gómez-Peralta MA, Imeldalacayo-Morales J \& Rosales-Rivera MC (2009) Hojas de chan (Hyptis suaveolens) para el control de Sitophilus zeamais y Zabrotes subfasciatus. Agronomía Mesoamericana 20: 263-273.

Grecco ED, Silveira LFV, Lima VLS \& Pezzopane JEM (2011) Estimativa do índice de área foliar e determinação do coeficiente de extinção luminosa da abóbora Cucurbita moschata var. japonesa. Idesia 29: 37-41.

Hinnah FD, Heldwein AB, Maldaner IC, Loose LH, Lucas DDP \& Bortoluzzi MP (2014) Estimativa da área foliar da berinjela em funções das dimensões foliares. Bragantia 73: 213-218.

Jadoski SO, Lopes ÉC, Maggi MF, Suchoronczek A, Saito LR \& Denega S (2012) Método de determinação da área foliar da cultivar de batata Ágata a partir de dimensões lineares. Semina 33: 2545-2554.

Janssen PHM \& Heuberger PSC (1995) Calibration of process - oriented models. Ecological Modelling 83: $55-56$.

Kumar R (2009) Calibration and validation of regression model for non-destructive leaf area estimation of saffron (Crocus sativus L.). Scientia Horticulturae 122: 142-145.

Leite HG \& Lima VCA (2002) Um método para condução de inventários florestais sem o uso de equações volumétricas. Revista Árvore 26: 321-328.

Leite MLMV, Lucena LRR, Sá Júnior EH \& Cruz MG (2017) Estimativa da área foliar em Urochloa mosambicensis por dimensões lineares. Revista Agropecuária Técnica 38: 9-16.

Lorenzi HE \& Matos FJA (2002) Plantas medicinais no Brasil: nativas e exóticas. Instituto Plantarum, Nova Odessa. 512p.

Lu HY, Lu CT, Wei ML \& Chan LF (2004) Comparison of different models for nondestructive leaf area estimation in taro. Agronomy Journal 96: 448-453.

Lucena LRR, Leite MLMV, Cruz MG \& Sá Júnior EH (2018) Estimativa da área foliar em Urochloa mosambicensis por dimensões foliares e imagens digitais. Archivos de Zootecnia 67: 408-413.

Lucena RRM, Batista TMV, Dombroski JLD, Lopes WAR \& Rodrigues GSO (2011) Medição de área foliar de aceroleira. Revista Caatinga 24: 40-45.

Maia SSS, Pinto JEBP, Silva FN \& Oliveira C (2008) Enraizamento de Hyptis suaveolens (L.) Poit. (Lamiaceae) em função da posição da estaca no ramo. Revista Brasileira de Ciências Agrárias 3: 317-320.

Maracajá PB, Madalena JÁ, Araújo E, Lima BG \& Linhares PC (2008) Estimativa de área foliar de juazeiro por dimensões lineares do limbo foliar. Revista Verde de Agroecologia 3: 1-5.
Oliveira PS, Silva W, Costa AAM, Schmildt ER \& Vitória EL (2017) Leaf area estimation in litchi by means of allometric relationships. Revista Brasileira de Fruticultura 39: 1-6.

Padrón RAR, Lopes SJ, Swarowsky A, Cerquera RR, Nogueira CU \& Maffei M (2016) Non-destructive models to estimate leaf area on bell pepper crop. Ciência Rural 46: 1938-1944.

Peksen E (2007) Non-destructive leaf area estimation model for faba bean (Vicia faba L.). Scientia Horticulturae 113: 322-328.

Pompelli MF, Antunes WC, Ferreira DTRG, Cavalcante PGS, Wanderley Filho HCL \& Endres L (2012) Allometric models for non-destructive leaf area estimation of Jatropha curcas. Biomass and Bioenergy 36: 77-85.

R Core Team (2018) R: a language and environment for statistical computing. R Foundation for Statistical Computing, Vienna. Disponível em $<$ https://www.rproject.org/>. Acesso em 3 junho 2018.

Raizada P (2006) Ecological and vegetative characteristics of a potent invader, Hyptis suaveolens Poit. from India. Lyonia 11: 115-120.

Ribeiro JES, Barbosa AJS \& Albuquerque MB (2018) Leaf Area Estimate of Erythroxylum simonis Plowman by Linear Dimensions. Floresta e Ambiente 25: 1-7.

Sachet MR, Penso GA, Pertille RH, Guerrezi MT \& Citadini I (2015) Non destructive leaf area estimation in peach tree. Ciência Rural 45: 21612163.

Satish V, Ravichandran VD, Usha G \& Paarakh MP (2010) Antimicrobial studies on the extracts of Cocculus hirsutus Linn. and Hyptis suaveolens Poit. Indian Journal of Natural Products and Resources 1: 49-52.

Schmildt ER, Amaral JAT, Santos JS \& Schmildt O (2015) Allometric model for estimating leaf area in clonal varieties of coffee (Coffea canephora). Revista Ciência Agronômica 46: 740-748.

Schmildt ER, Hueso JJ \& Cuevas J (2014) Allometric models for determining leaf area of vine 'Sugraone'. Ciência e Técnica Vitivinícola 29: 61-81.

Schmildt ER, Negris FS, Cesana Júnior E, Schmildt O \& Alexandre RS (2016a) Equações para estimar área foliar de maracujá amarelo. Nucleus 13: 97-104.

Schmildt ER, Oliari LS, Schmildt O, Alexandre RS, Brumatti JA \& Viana DG (2016b) Determinação da área foliar de macadâmia a partir de dimensões lineares do limbo foliar. Revista Agro@mbiente On-line 10: 209-216.

Silva SF, Pereira LR, Cabanez PA, Mendonça RF \& Amaral JAT (2017) Modelos alométricos para estimativa da área foliar de boldo pelo método não destrutivo. Agrarian 10: 193-198.

Sousa LF, Santos JGD, Alexandrino E, Maurício RM, Martins AD \& Sousa JTL (2015) Método prático e eficiente para estimar a área foliar de gramíneas 
forrageiras tropicais. Archivos de Zootecnia 64: 83-85.

Taiz L, Zeiger E, Møller IM \& Murphy A (2017) Fisiologia e desenvolvimento vegetal. $6^{\mathrm{a}}$ ed. Artmed, Porto Alegre. 888p.

Tartaglia FL, Righi EZ, Rocha L, Loose LH, Maldaner IC \& Heldwein AB (2016) Non-destructive models for leaf area determination in canola. Revista Brasileira de Engenharia Agrícola e Ambiental 20: 551-556.

Toebe M, Cargnelutti Filho A, Loose LH, Heldwein AB \& Zanon AJ (2012) Leaf area of snap bean (Phaseolus vulgaris L.) according to leaf dimensions. Semina 33: 2491-2500.

Willmott CJ, Ackleson SG, Davis RE, Feddema JJ, Klink KM, Legates DR, O’Donnell J \& Roew CM (1985) Statistics for the evaluation and comparison of models. Journal of Geophysical Research 90: 8995-9005.

Xue Q, Weiss A \& Baenziger PS (2004) Predicting leaf appearance in field-grown winter wheat: evaluating linear and non-linear models. Ecological Modelling 175: 261-270. 